\title{
Pattern of Musculoskeletal Injuries Amongst Male Amateur Basketball Players in Anambra State, Nigeria
}

\author{
Christian Arinze Okonkwo, MSc ${ }^{1^{*}}$, Eustus Chigemezu Okereke, B.MR ${ }^{1}$, Joseph Onuwa \\ Umunnah, Ph.D. ${ }^{1}$, Peter Olanrewaju Ibikunle, Ph.D. ${ }^{1}$, Victor Egwuonwu, Ph.D. ${ }^{1}$, \\ Stanley Maduagwu, Ph.D. ${ }^{1}$, Kenneth Umezurike Ani, MSc ${ }^{1}$, Dickson Obinna Nwodo, \\ $\mathrm{MSc}^{2}$, Samuel Chidile Muonwe, MSc ${ }^{1}$ and Chiamaka Ann Nwanne MSc ${ }^{1}$
}

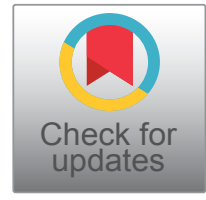

\author{
${ }^{1}$ Medical Rehabilitation Department, Faculty of Health Sciences and Technology, Nnamdi Azikiwe University, Nigeria \\ ${ }^{2}$ Department of Physiotherapy, Federal Teaching Hospital Abakiliki, Nigeria
}

*Corresponding author: Christian Arinze Okonkwo, MSc, Department of Medical Rehabilitation, Faculty of Health Sciences and Technology, College of Health Sciences, Nnamdi Azikiwe University, Nnewi Campus, Nigeria, Tel: +2348064137839

\begin{abstract}
Background: Musculoskeletal Injuries (MSIs) are expected consequences of participation in sports-related occupations like basketball given that it is a contact sport with complex movements that include jumps, turns, and quick changes in direction.

Aim: This study, therefore, explored the pattern of MSIs among amateur basketball players in the Anambra State of Nigeria and the correlation between socio-demographic variables and MSIs.
\end{abstract}

Methods: A cross-sectional survey research design was used for this study. One hundred and twelve (112) consenting male basketball players were consecutively recruited for this study. Socio-demographic data was obtained and recorded. Cornell Musculoskeletal Discomfort Questionnaire (CMDQ) was used to evaluate MSIs among the participants.

Results: The injury proportions of the participants were; the right knee $(40.27 \%)$, left knee $(34.96 \%)$, right foot $(6.06 \%)$, left foot $(3.96 \%)$, right shoulder $(3.64 \%)$, right wrist $(83.60 \%)$ and left wrist $(2.93 \%)$, lower back $(0.99 \%)$, Left thigh $(0.92 \%)$, Left lower leg $(0.88 \%)$, right thigh $(0.71 \%)$. MSI had significant moderate correlation with height $(r=0.381, p<0.01)$, weight $(r=0.431, p<0.01)$ and significant moderate correlation with hours of training per day $(r=0.513, p<0.01)$. However, there was no significant correlation between MSIs and Body Mass Index $(r=0.037$, $p=0.067)$ and between $\mathrm{MSI}$ and age $(r=-0.132, p=0.167)$.
Conclusion: The pattern of MSls amongst male amateur basketball players are right knee, left knee, left foot, right foot, right shoulder, right wrist, left wrist, etc. Height, weight, and hours of training per day correlated significantly with MSIs. Age and Body Mass Index (BMI) did not correlate significantly with MSIs. Basketball players should therefore be encouraged to use protective equipment for these parts and physical therapy may help in the prevention and management of MSIs among basketball players.

\section{Keywords}

Musculoskeletal injuries, Amateur, Male basketball players

\section{Introduction}

\section{Background of the study}

Musculoskeletal Injuries (MSIs) are injuries that affect the human body's movement or musculoskeletal system (i.e. muscles, tendons, ligaments, nerves, discs, blood vessels, etc.). Musculoskeletal injuries usually occur when there is an overload of the musculoskeletal structures that exceed the ability of regeneration or adaptation [1]. Common musculoskeletal injuries include tendonitis, muscle/tendon strain, ligament sprain, tension neck syndrome, rotator cuff tendonitis, epicondylitis, and ruptured/herniated disc [2].

MSIs are expected consequences of participation in sports-related occupations like basketball given that it 
is a contact sport with complex movements that include jumps, turns, and quick changes in direction. Sports injury is a public health concern, as it has detrimental effects on the health and well-being of athletes [1].

MSI, generally, is not caused as a result of a single causative factor but is associated with various risk factors interacting at a given time [3]. Such risk factors have been classified into "extrinsic" and "intrinsic factors", with the extrinsic factors playing a dominant role. Intrinsic factors were defined as factors associated with the athletes' characteristics, such as anthropometric measurements (example, joint laxity, limb alignment, notch dimension, ligament size, and body weight), nutrition, use of drugs (example; alcohol and smoking), use of performance enhancers (example, steroids) and psychological factors [4]. Extrinsic factors, on the other hand, were defined as factors related to the environment, climate, equipment, training (which include overtraining, lack of rest), and sports characteristics (the movements inherent to the particular sport) [4].

The various types of basketball injuries in the order of their prevalence are sprain (52.7\%), bruises (32.7\%), dislocation (29.1\%), cram (18.2\%), strain (10.9\%), fracture (10\%), and laceration (3.6\%), concussion (0.9\%) [5]. Per body region, the prevalence of MSIs was investigated by Andreoli, et al. [6], in their systemic review which comprised of more than 12,000 basketball injuries presented in works from various professional basketball-playing countries. It showed that there were generally more injuries in the lower limbs than any other body region, regardless of gender. The largest proportion of injuries occurred in the ankle (21.9\%), followed by the knee (17.8\%). Many authors have pointed to the ankle as the most common site of injury [7-10]. With regards to upper limb injuries, injuries to the hands, fingers, and wrists (8.7\%) predominated over the shoulder, arm, and forearm injuries (4.5\%). Injuries that occurred in the trunk and spine represent $7.5 \%$ of all injuries. The work of Akodu, et al. [1], on a Nigerian professional basketball-playing population specifically, showed a similar pattern of injuries with most injuries being recorded in the lower limb, upper limb, and trunk in that order.

Epidemiological studies have also revealed that male basketball players are more prone to injuries than their female counterparts; a finding that has been attributed to greater physical exertion among males than females during play [6]. Also, psychological conditioning and drug use were identified as major factors as males played with a greater competitive spirit and had a greater prevalence of drug use [6]. Studies have also shown that there was a higher incidence of injuries in Nigeria professional basketball players than other populations, a finding that has been linked mainly with extrinsic factors like poor playing courts made of concrete, and other inadequate playing facilities [11].
With basketball continuing to gain popularity in Nigeria coupled with increased participation at all levels of play, from recreational, armature to professional, there is a need for assessment of the pattern of injuries related to this sport at the armature level. Unfortunately, there is still a dearth of empirical data on the patterns of basketball-related MSIs among amateur players in any of the South-Eastern Nigerian States. This study was therefore aimed to establish the pattern of MSIs among armature basketball players in the Anambra State of Nigeria, and the relationship between age, height, body mass index, playing hours per day on the MSIs.

\section{Materials and Methods}

\section{Study design and sample}

The study was a cross-sectional survey involving armature basketball players. They were consecutively recruited from eleven conveniently selected armature basketball-playing communities in Anambra State, Nigeria, whose total population was one hundred and fifty-six (156). The Ethics Committee of Nnamdi Azikiwe University Teaching Hospital, Nnewi, Anambra State gave approval for the study, and all participants gave their informed consent after the purpose and procedure for the study had been explained to them. Participants for this study were amateur basketball players who have been actively competing or training for a minimum of one year in Anambra State. Amateur basketball players who have not been actively competing or training for a minimum of one year and those involved in any other kind of sports-related occupation like football, volleyball, tennis, boxing, rugby, cricket, etc were excluded from the study. A sample size of 112 was obtained from the total population using the formula suggested by Taro Yamane [12]. The socio-demographic variables (age, height, weight, body mass index, years of playing, hours of playing per day) were recorded.

$$
\begin{aligned}
& n=\frac{N}{1+N(e)^{2}} \\
& \text { Where, } \\
& \mathrm{n}=\text { corrected sample size } \\
& \mathrm{N}=\text { population size } \\
& \mathrm{e}=\text { Margin of Error (MoE), } \mathrm{e}=0.05
\end{aligned}
$$

\section{Measurements}

Height: was measured in centimeters using a stadiometer. Participants are asked to remove shoes, heavy outer garments, stands with their back to the height rule, the back of the head, back, buttocks, calves, and heels touch the upright rule and feet together. The top of the external ear canal was leveled with the inferior margin of the cheekbone and the participant looked straight. The headpiece of the stadiometer was lowered so that the hair (if present) is pressed flat. The value was obtained and recorded. 
Table 1: Socio-demographic profile of the participants.

\begin{tabular}{|l|l|l|l|l|}
\hline Variables & N & Minimum & Maximum & Mean \pm STD \\
\hline Age (Years) & 112 & 18 & 36 & $25.43 \pm 4.66$ \\
\hline Height $(\mathrm{Cm})$ & 112 & 158 & 192 & $174.98 \pm 7.89$ \\
\hline Weight $(\mathrm{Kg})$ & 112 & 50 & 100 & $70.22 \pm 11.12$ \\
\hline BMl $\left(\mathrm{Kg} / \mathrm{M}^{2}\right)$ & 112 & 17.71 & 29.41 & $22.70 \pm 2.81$ \\
\hline Years of playing (Years) & 112 & 1 & 18 & $4.13 \pm 3.11$ \\
\hline Duration of play (Hours) & 112 & 1 & 5 & $2.86 \pm 1.34$ \\
\hline
\end{tabular}

Key: BMI means body mass index

Body weight: was measured in kilograms using a weighing scale. The participants were asked to remove their footwear and heavy outer garments or objects from their bodies. The participant stood on the footpad with the weight evenly distributed on both feet. The weight was taken by bending over the scale and the reading was recorded.

Body Mass Index (BMI) in $\mathrm{Kg} / \mathrm{M}^{2}$ : This was calculated by dividing the weight of each participant by the square of his height. Usually, an individual with a BMI of 16 to 18.5 is considered underweight, 18.5 to 25 is said to be normal, 25 to 30 is considered overweight and 30 to 35 is obese. An individual with a BMI below 16 is considered severely underweight while any BMI above 35 is said to be severely obese.

The pattern of musculoskeletal injuries was determined using an instrument called Cornell Musculoskeletal Discomfort Questionnaire (CMDQ) developed by Professor Allan Hedge [13], at Cornell University. The CMDQ is an 11-item self-report and researcher-administered standardized questionnaire which assesses occupational-related musculoskeletal ache, pain, and discomfort at different points of the body such as the neck, shoulder, upper back, upper arm, lower back, forearm, wrist, buttocks, thigh, knee, and lower leg. They were assessed in terms of frequency, discomfort, and interference with work. The scores for musculoskeletal injuries were calculated following the CMDQ scoring guidelines for determining the quantification of frequency, discomfort, and interference [13]. The response to the frequency of musculoskeletal injury was weighed on 6 points scale ranging from 0 to 10 ( $0=$ Never; $1-2$ times $/$ week $=1.5$; 3-4 times/week-3.5; Everyday $=5$, and 10 for several times/day). Different weighing categories (1 for slight, 2 for moderate, 3 for very) were used to quantify responses for each body discomfort. For each body interference; Not was weighed 1, Slight was weighed 2, substantial was weighed 3 . For each MSI identified by each participant in any part of the body, we multiplied each weighing category assigned, by the number of responses $(n)$ for each body part. The outcomes of each multiplication were summed up to generate an overall score for frequency, discomfort, and interference respectively. The test-retest reliability for $C M D Q$ was in the moderate to a substantial range of 0.41-0.80 [13].

\section{Data analysis}

The data obtained was inputted in Statistical Package for Social Science (SPSS) version 20. The full description of the participants was summarized using mean and standard deviation. The pattern of injuries was presented using frequency and percentages. Pearson's correlation and Spearman's correlation test were used to establish the relationship between MSIs and age, height, weight, $\mathrm{BMI}$, and playing hours per day. Alpha level was set at 0.05 .

\section{Results}

The participants were aged between 18 and 36 years with a mean age of $25.43 \pm 4.66$ years. The years of playing basketball ranged from 1 to 18 years with average years being $4.13 \pm 3.11$ years. The duration of playing per day ranged between 1 hour and 5 hours. Their average height, weight, and Body Mass Index (BMI) were $174.98 \pm 7.89 \mathrm{~cm}, 70.22 \pm 11.12 \mathrm{~kg}$, and $22.70 \pm 2.81 \mathrm{Kg} / \mathrm{M}^{2}$ respectively (Table 1 ). The injury proportions of the participants were; the right knee (40.27\%), left knee (34.96\%), right foot (6.06\%), left foot (3.96\%), right shoulder (3.64\%), right wrist $(83.60 \%)$ and left wrist $(2.93 \%)$, lower back (0.99\%), Left thigh (0.92\%), Left lower leg $(0.88 \%)$, right thigh $(0.71 \%)$, left shoulder (0.49\%) (Table 2a and Table 2b). From the distribution in Table 2a, The responses ( $n$ ) to the frequency of MSIs were weighed on 6 points scale ranging from 0 to 10 (0 = Never; 1 -2 times/week = 1.5; 3-4 times/week-3.5; Everyday $=5$, and 10 for several times /day). Different weighing categories (1 for slight, 2 for moderate, 3 for very) were used to quantify responses for each body discomfort. For each body interference; Not was weighed 1 , Slight was weighed 2, substantial was weighed 3. For each MSI identified by each participant in any part of the body, we multiplied each weighing category assigned, by the number of responses ( $n$ ) for each body part. The outcomes of each multiplication were summed up to generate an overall score for frequency, discomfort, and interference respectively (Table 2b). The percentage contribution of each body part to the total score was then calculated (Table $2 b$ ). The right knee $(40.27 \%)$ and the left knee (34.86\%) were the major contributors to the MSIs score (Table 2b). Significant positive correlation was found between MSIs and height $(r=0.381, p<0.01), M S I$ and weight $(r$ 


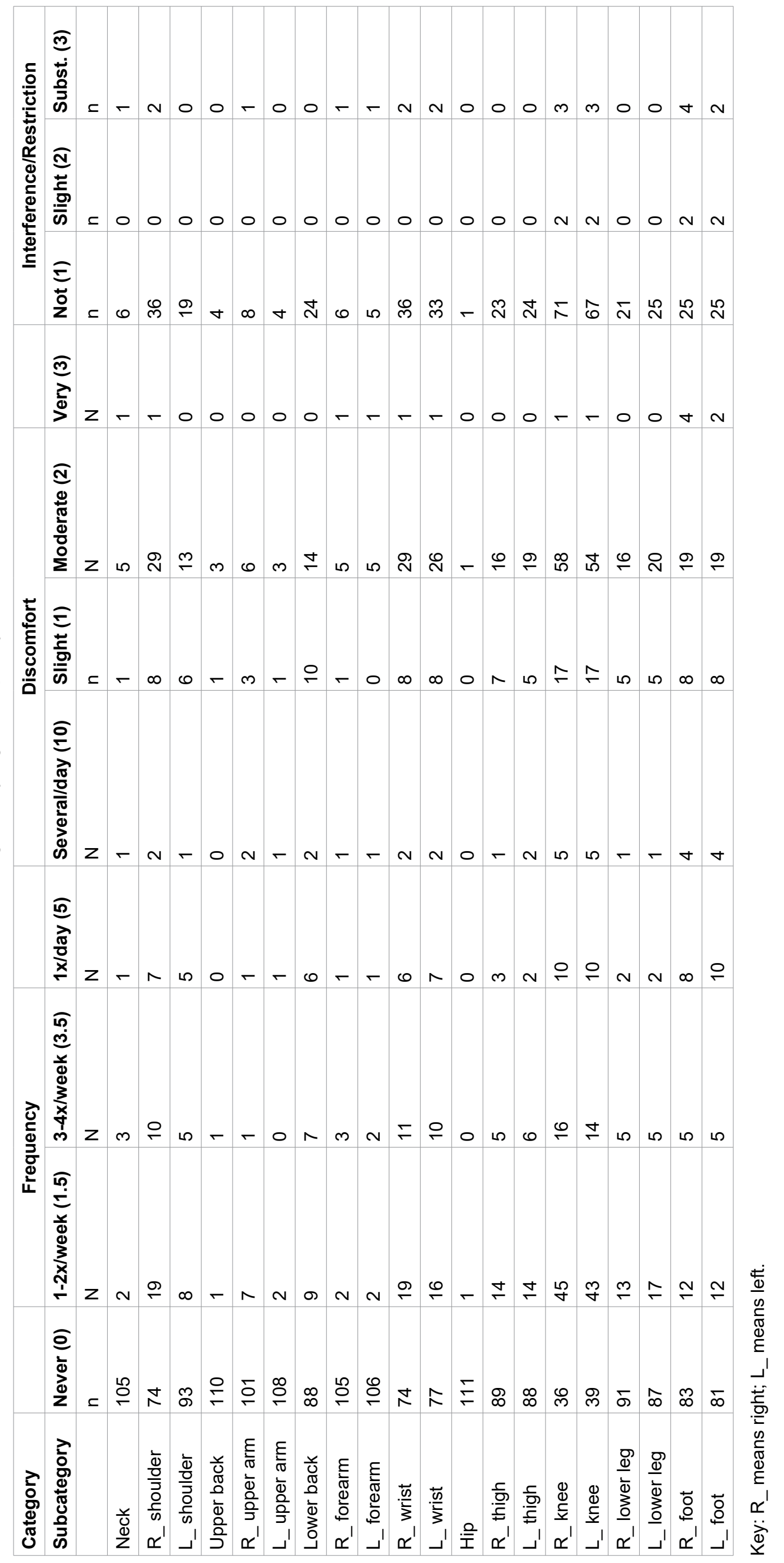


Table 2b: Distribution of MSI according to frequency score, discomfort score, and interference score.

\begin{tabular}{|c|c|c|c|c|c|}
\hline Part & Frequency & Discomfort & Interference & Score & $\%$ total \\
\hline Neck & 28.5 & 14 & 6 & 2394 & 0.03 \\
\hline R_shoulder & 118.5 & 69 & 36 & 294354 & 3.64 \\
\hline L_ shoulder & 64.5 & 32 & 19 & 39216 & 0.49 \\
\hline Upper back & 5 & 7 & 4 & 140 & 0.00 \\
\hline R_upper arm & 39 & 15 & 8 & 4680 & 0.06 \\
\hline$L_{-}$upper arm & 18 & 7 & 4 & 504 & 0.01 \\
\hline Lower back & 88 & 38 & 24 & 80256 & 0.99 \\
\hline R_forearm & 28.5 & 14 & 6 & 2394 & 0.03 \\
\hline$L_{-}$forearm & 25 & 13 & 5 & 1625 & 0.02 \\
\hline R_wrist & 117 & 69 & 36 & 290628 & 3.60 \\
\hline$L_{\text {_ wrist }}$ & 114 & 63 & 33 & 237006 & 2.93 \\
\hline Hip & 1.5 & 2 & 1 & 3 & 0.00 \\
\hline$R_{\text {_thigh }}$ & 63.5 & 39 & 23 & 56959.5 & 0.71 \\
\hline$L_{-}$thigh & 72 & 43 & 24 & 74304 & 0.92 \\
\hline R_knee & 223.5 & 136 & 107 & 3252372 & 40.27 \\
\hline$L_{\text {_ knee }}$ & 213.5 & 128 & 103 & 2814784 & 34.86 \\
\hline$R_{\text {_ l lower leg }}$ & 57 & 37 & 21 & 44289 & 0.55 \\
\hline L_ lower leg & 63 & 45 & 25 & 70875 & 0.88 \\
\hline R_foot & 115.5 & 58 & 73 & 489027 & 6.06 \\
\hline$L_{-}$foot & 125.5 & 52 & 49 & 319774 & 3.96 \\
\hline Total & & & & 8075585 & 100.00 \\
\hline
\end{tabular}

Key: R_means right; L_ means left.

Table 3: Correlation between MSIs and Age, Height, Weight, BMI, and Duration of training per day.

\begin{tabular}{|l|l|l|l|l|l|l|}
\hline & & AGE & HEIGHT & WEIGHT & BMI & DURATION PER DAY \\
\hline & & & & & & \\
\hline Total MSI & R & -0.132 & $0.381^{* *}$ & $0.431^{* *}$ & 0.037 & $0.513^{* *}$ \\
\hline & P & 0.167 & $<0.01$ & $<0.01$ & 0.697 & $<0.01$ \\
\hline & N & 112 & 112 & 112 & 112 & 112 \\
\hline
\end{tabular}

Key: MSI means Musculoskeletal Injuries.

$=0.431, p<0.01)$, MSIs and hours of training per day $(r$ $=0.513, p<0.01)$. The correlation between MSIs and BMI was weak and insignificant ( $r=0.037, p<0.697)$. The correlation between MSIs and age was negative and insignificant ( $r=-0.132, p=0.167)$ (Table 3).

\section{Discussion}

Athletes are known to be at higher risk of musculoskeletal injuries $[14,15]$. Such injuries result in musculoskeletal discomfort which may be significant and restrict function [14-16]. This study was aimed to evaluate the pattern of musculoskeletal discomfort among amateur basketball players in Anambra state, Nigeria.

As seen from the findings, the average age and height of amateur basketball players were $25.43 \pm 4.66$ years and $174.98 \pm 7.89 \mathrm{~m}$ respectively. These findings are similar to those of a previous study in a SouthWestern Nigerian State by Akodu [1]. These indicate that becoming an amateur basketball player requires proportionate traits like age and heights of players across states, and maybe a preparatory requirement to become a professional player [17]. Brian [18] suggested that taller lower limbs are especially advantageous as players can cover more grounds and are closer to the basketball hoop.

Results from this study show that the lower limb was the most common site of discomfort amongst the study population; with the knee accounting for the highest percentage of discomfort followed by the foot. This pattern is similar to the recent findings by Mendhe and Borkar, [19], whose result, showed that there were more injuries in lower limbs $60.2 \%$ of which the knee accounted for $22.8 \%$ and the ankle accounted for $19.4 \%$. Legault [20] also observed that lower extremities and lower back symptoms were common conditions in the athletic adolescent population. In their work, Legault [20], compared discomfort in the control to an athletic 
group, and controls were found to have a significantly higher prevalence of symptoms affecting the spinal regions including the neck, the upper back, and the lower back. Furthermore, Akinbo [21] who studied professional basketball players in Nigeria found that the knee was second only to ankles as an area mostly injured in basketball. The suggested mechanism for foot injury involves impact with a floor of a basketball court, especially during jumping and landing on side of a foot or someone else's foot [19]. Similarly, Chong [22] also suggested that the mechanism of knee injury and discomfort may be by the maximal ground reaction force, in which structures of the knee are fully tensed by the impact load. In the upper limb, the shoulders, especially the right shoulder and the wrists were the areas with the most discomfort. While we reported a value of about $3.60 \%$ on either wrist, Mendhe and Borkar [19], reported $10.9 \%$ for wrist discomfort; Akinbo [21] reported a lower value of $5.1 \%$ similar to our findings. The most common factor in the occurrence of injury and discomfort to the wrist was said to be by falling on an outstretched hand [23]. Also, injury of the shoulder/ arm was said to be mostly caused by frequent overhead movements while shooting the ball or while passing it to the player [21]. Neck injuries accounted for $0.03 \%$ of MSI in this study. Mendhe and Borkar, [21], reported discomfort in head and neck prevalence of $10.5 \%$. Poor posture and techniques of play may have accounted for the wide gap in the latter. This can be buttressed by the fact that the authors stated that the injury mechanism involved bumping of heads with another player or accidental hit by the elbow or ball. Low back pain was also a prevalent complaint in a studied population, with rates reaching 20 to $50 \%$ during adolescence $[24,25]$. Another study reported similar results, with a prevalence of $45.1 \%$ for low back symptoms [15].

Hours of training showed a significant correlation with MSIs. Mendhe and Borkar, [19], had earlier made a similar suggestion, saying that less frequency of matches and increased training sessions can also lead to more injuries during the training sessions. But few studies reported more injuries at the competitive level: as a report in one study showed higher rates of injury during competition may be due to increased play intensity, increased legal, illegal physical contact also due to increased exposure to high-risk activities [26]. A study found that ankle injuries were the most common type of injuries but that knee injury accounted for the greatest number of games missed [27]. Age did not show a significant correlation with MSIs. This is similar to the findings of Legault [20], who observed that age did not play a significant role in the relationship between musculoskeletal symptoms prevalence/impacts on adolescents. Weight and height showed a significant correlation but not BMI. It appears that high BMI in this population was due to lean body mass, which translates to a stronger build and lesser chances of injury and
MSIs. On the other hand, increased height and weight have been identified as risk factors for developing MSI [28-30]. Of these factors, weight is a readily modifiable factor and therefore should be a target in the attempt to prevent and control MSIs.

\section{Conclusion}

Based on the findings of the study: The pattern of MSIs amongst male amateur basketball players are right knee, left knee, left foot, right foot, right shoulder, right wrist, left wrist, etc. Height, weight, and duration of training per day correlated significantly with MSI. Age and Body Mass Index did not correlate significantly with MSI.

\section{Recommendations}

Based on the findings presented in this research, the researchers recommend that protective wear should be used by amateur basketball players to prevent sports injury and reduce MSIs. Amateur basketball players should balance training with rest to prevent overuseinduced MSIs.

\section{Limitations}

High insecurity at the time of research made traveling to collect data a bit challenging.

\section{Acknowledgment}

The authors wished to thank the management of various armature basketball playing teams across Anambra State, Nigeria for their support in ensuring that this study was eventful.

\section{Author Contributions}

CAO was involved in the conception, design, analysis, drafting, and revision of the manuscript. ECO was involved in data collection and literature review. JOU was involved in the conception, design, analysis, drafting, and revision of the manuscript. POI was involved in the design, drafting, and revision of the manuscript. VE was involved in the drafting and revision of the manuscript. SM was involved in the conception, design, analysis, drafting, and revision of the manuscript. KUM was involved in the conception, design, analysis, drafting, and revision of the manuscript. DON was involved in data collection and Literature review. SCM was involved in the conception, design, analysis, drafting, and revision of the manuscript. CAN was involved in the conception, design, and literature review.

\section{Ethical Approval}

All procedures performed in studies involving human participants were done following the ethical standards of the institutional and/or national research committee and with the 1964 Helsinki declaration and its later amendments or comparable ethical standards.

\section{Funding}

Self-funded. 


\section{Informed Consent}

Informed consent was obtained from all individual participants included in the study.

\section{Conflict of Interest}

The authors declare that they have no conflict of interest.

\section{References}

1. Akodu AK, Akinbo SRA, Ajiboye AR (2017) Injury patterns and perceived risk factors among basketball players in Nigeria. Medicina Sportiva 13: 2918-2926.

2. Carlos VC, Barbara CC, Elisabeth B, Alberto CP, Benno E, et al. (2018) Epidemiology of sports injuries in basketball: An integrative systematic review. BMJSEM 4.

3. Saragiotto BT, Carla DP, Alexandre DL (2014) Risk factors and injury prevention in elite athletes: A descriptive study of the opinions of physical therapists, doctors, and trainers. Brazilian Journal of Physical Therapy 18: 137-143.

4. Meeuwisse WH, Sellmer R, Hagel BE (2002) Rates and risks of injury during intercollegiate basketball. American Journal of Sports Medicine 31: 379-385.

5. Ayanniyi O, Oluwasegun AB, Babatunde AA (2015) The pattern of musculoskeletal injuries among soccer and basketball players in a Nigerian university. Medicina Sportiva 11: 2676-2681.

6. Andreoli O, Abiodun BO, Adekanla BA (2015) The pattern of musculoskeletal injuries among soccer and basketball players in a Nigerian University. Medicina Sportiva 11: 2676-2681.

7. Dick R, Hertel J, Age J, Grossman J, Marshall SW (2007) Descriptive epidemiology of collegiate men's basketball injuries: National collegiate athletic association injury surveillance system, 1988-1909 through 2003-2004. J Athl Train 42: 194-201.

8. Agel J, Olson DE, Dick R (2007) Descriptive epidemiology of collegiate women's basketball injuries: National Collegiate Athletic Association Injury Surveillance System. J Athl Train 42: 202-210.

9. Starkey C (2000) Injuries and illnesses in the national basketball association: A 10-year perspective. J Athl Train 35: 161-167.

10. Zelisko JA, Noble HB, Porter M (1982) A comparison of men's and women's professional basketball injuries. Am J Sports Med 10: 297-299.

11. Owoeye OBA, Akodu AK, Oladokun BM (2012) Incidence and pattern of injuries among adolescent basketball players in Nigeria. Sports Med, Arthrosc, Rehabil Ther Technol 4: 4-15.

12. Taro Y (1976) Statistics: An introductory analysis. (2nd edn), New York: Harper and Row Publishers.

13. Hedge A, Morimoto S, Mccrobie D (1999) Effects of Keyboard tray geometry on upper body posture and comfort. Ergonomics 42: 1333-1349.
14. Caine D, Caine C, Maffulli N (2006) Incidence and distribution of pediatric sports-related injuries. Clin J Sport Med 16: 500-513.

15. Emery CA, Meeuwisse WH, McAllister JR (2006) Survey of sport participation and sports injury in Calgary and area high schools. Clin J Sport Med 16: 20-26.

16. Kalbrosky B (2020) NBA Draft Big Board 5.0: Final Update Ranking Top 100 Prospects. The rookie wire.

17. (2010) FIBA Official Basketball Rules. Rule 4, Section 8.1.

18. Brian M (2017) Why Do Basketball Hoops Have Nets? Basketball World.

19. Mendhe S, Borkar S (2021) Epidemiology of musculoskeletal injuries in basketball players: Systematic review." International Journal of Physical Education, Sports and Health 8: 111-116.

20. LegaultÉP, Descarreaux M, Cantin V (2015)Musculoskeletal symptoms in an adolescent athlete population: a comparative study. BMC Musculoskelet Disord 16: 210.

21. Akinbo SD, Odebiyi E, Adebayo A (2008) The pattern of musculoskeletal injuries in professional basketball league in Nigeria. The internet journal of rheumatology 5: 87-88.

22. Chong D, Chan A (2010) Time-motion analysis of Italian elite women's basketball games: Individual and team analyses. J Strength Cond Res 29: 144-150.

23. Stögner VA, Kaltenborn A, Laser H, Vogt PM (2020) "Hand injuries in sports - a retrospective analysis of 364 cases". BMC Musculoskeletal Disorders 21: 826.

24. Kjaer P, Wedderkopp N, Korsholm L, Leboeuf-Yde C (2011) Prevalence and tracking of back pain from childhood to adolescence". BMC Musculoskelet Disord 12: 98.

25. Masiero S, Carraro E, Celia A, Sarto D, Ermani M (2014) Prevalence of nonspecific low back pain in schoolchildren aged between 13 and 15 years. BMC Paediatrics 97: 212-216.

26. Sreekaarini I, Eapen C, Zulfeequer CP (2014) Prevalence of sports injuries in adolescent athletes. J Athl Enhancement 5: 2.

27. DiStefano LJ, Dann CL, Chang CJ, Putukian M, Pierpoint LA, et al. (2018) The first decade of web-based sports injury surveillance: descriptive epidemiology of injuries in US high school girls' soccer (2005-2006 through 2013-2014) and national collegiate athletic association women's soccer (2004-2005 through 2013-2014). J Athl Train 53: 880-892.

28. Willem HM, Hugh T, Brent H, Carolyn E (2007) A dynamic model of etiology in sports injury: the recursive nature of risk and causation. Clin J Sports Med 17: 215-219.

29. Abaraogu UO, Okafor C, Ezeukwu AO, Igwe SE (2015) Prevalence of work-related musculoskeletal discomfort and its impact on activity: A Survey of Beverage Factory Workers in Eastern Nigeria. Journal of Work 52: 627-634.

30. Tamene A, Mulugeta H, Ashenafi T, Thygerson SM (2020) Musculoskeletal Disorders and Associated Factors among Vehicle Repair Workers in Hawassa City, Southern Ethiopia. Journal of Environmental and Public Health 2020: 9472357. 